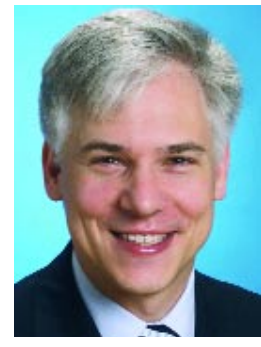

Jürgen Bauer

\title{
Ernährung und Demenz
}

\author{
Jürgen Bauer und Cornel Sieber
}

Lehrstuhl für Innere Medizin V, Universität Erlangen-Nürnberg

Medizinische Klinik 2 - Schwerpunkt Geriatrie, Klinikum Nürnberg

psychoneuro 2004; 30 (9): 481-488

D ie Altersmedizin nimmt aufgrund der demographischen Entwicklung rasch an Aktualität zu. Die Menschen werden immer älter, und dies bei besserer $\mathrm{Ge}-$ sundheit. So hat sich die durchschnittliche Lebenserwartung der Amerikaner im 20. Jahrhundert um knapp zwei Drittel erhöht. Wenn auch versetzt, so ist der rapide Anstieg der durchschnittlichen Lebenserwartung auch in den sogenannten Schwellenländern (z.B. Brasilien, Indien, Mexiko und China) zu beobachten. In diesen Ländern ist die Lebenserwartung von gut 40 Jahren 1950 auf knapp 65 Jahre im Jahre 2000 gestiegen.

In Deutschland wird sich zwischen 2002-2020 die Anzahl der über 80-jährigen Männer verdreifachen. Die über 80-Jährigen sind auch die am raschesten zunehmende Bevölkerungsgruppe in Deutschland. Im 2002 erschienenen „vierten Bericht zu Lage der älteren Generation in der Bundesrepublik Deutschland“ werden folgende Zahlen genannt: 2002 lebten in Deutschland 2,9 Millionen Menschen, die über 80 Jahre alt waren (3,6\% der Bevölkerung). Im Jahre 2020 werden dies 5,1 Millionen Personen sein (6,3\%). Glaubt man an die Vorausberechnungen für das Jahr 2050, so werden dies 8,0 Millionen Menschen sein. Demenzkrankheiten sind klar mit zunehmendem Lebensalter korreliert, mit einer Inzidenz von zirka $30 \%$ bei den über 85-Jährigen. Somit wird auch die

Die Anzahl von Demenzkranken in Deutschland wird im Rahmen der allgemeinen demographischen Entwicklung in den nächsten Jahrzehnten deutlich zunehmen. Eines der drängendsten Probleme in der Betreuung dieser Patienten ist das der angemessenen Ernährung in den verschiedenen Krankheitsstadien. Es geht hierbei zum einen um die Vermeidung einer frühzeitigen Mangelernährung, welche zusätzliche funktionelle Einbußen bedingen kann. Zum anderen gilt es auch, eine Übertherapie in späten Stadien der Erkrankung zu vermeiden. Demente Patienten sollten in frühen und mittleren Krankheitsstadien auf das Vorliegen einer Mangelernährung gescreent werden. Für durch eine Malnutrition gefährdete Patienten sollten in diesen Stadien inidviduelle Ernährungspläne erstellt werden. Die Einleitung einer enteralen Ernährung in fortgeschrittenen Demenzstadien sollte jeweils Folge einer sorgfältigen Einzelfallentscheidung sein.

(Mangel-) Ernährung - eines der häufigsten Begleitprobleme bei Demenzkranken - ein rasch sich akzentuierendes Problem darstellen.

Vor allem in Pflegeheimen ist der Anteil von Patienten mit Demenzerkrankungen sehr hoch, nicht selten auch der Grund zur Einweisung (4). Zirka $80 \%$ aller Bewohner von Pflegeheimen haben ein psychiatrisches Leiden, wovon zwei Drittel Demenzleiden sind (25). Viele dieser Patienten weisen Zeichen einer Malnutrition, d.h. Fehlernährung auf.

Depressionen sind ebenfalls sehr häufig bei dementen Menschen, aber leider nur zu oft nicht diagnostiziert. Man kann davon ausgehen, dass nur $6-10 \%$ der dementen $\mathrm{Pa}$ tienten in Langzeitpflegestrukturen Antidepressiva erhalten $(6,17)$. Ein verminderter Appetit ist bei Dementen oft ein Zeichen für eine Depression (19).

Eine Malnutrition ist schon in Frühstadien von Demenzkranken häufig vorhanden und die Nahrungszufuhr wird zu einer der
Hauptpflegeaufgaben bei schwer Dementen. Es ergibt sich darüber hinaus ein großer Bedarf an Betreuungspersonen. Diese lassen sich aufgrund der niedrigen Natalität nicht mehr im eigenen Lande finden. Auch aus den neuen Bundesländern sowie aus den Ländern, die durch die Osterweiterung neu Teile der EU sind, werden sie wohl nicht rekrutiert werden können. So werden es wohl Pflegepersonen aus fernen Kulturen sein, die Demenzkranke betreuen werden, wie wir es schon jetzt immer mehr in der Kleinkinderbetreuung sehen. Dieser sogenannte „care drain“ stellt für diese Länder eine Problematik für sich dar. Auch sind Sprachbarrieren gerade bei der Betreuung Dementer ein Hindernis. Die Problematik einer adäquaten Ernährung bei Demenzkranken zeigt somit archtetypisch auf, wie wenig wir noch auf die Pflegeaufgaben, die in einer noch nie dagewesenen Geschwindigkeit auf uns zukommen, vorbereitet sind. 


\section{Tab. 1 Parameter zur Erfassung einer Mangelernährung bei Dementen}

- Body Mass Index

Körpergewicht

(Untergewicht $<20 \mathrm{~kg} / \mathrm{m}^{2}$ )

- Albumin

- Screening-/Assessment-

Verfahren pathologisch < 3,5 gr/dl

z.B. Mini Nutritional Assessment - MNA
Körperoberfläche ${ }^{2}$

\section{Diagnostik der Mangel- ernährung bei Dementen}

Wichtig ist, sich frühzeitig mit der Ernährungssituation dementer Patienten zu beschäftigen, um die Entwicklung fortgeschrittener Stadien der Mangelernährung zu verzögern, da diese regelhaft mit zusätzlichen funktionellen Einbußen einhergehen (Tab. 1) (30).

Die Beurteilung des Ernährungszustandes sollte mit dem Wiegen des Patienten beginnen. Bedeutsamer als das absolute Gewicht ist jedoch die Gewichtsentwicklung. Für diese ist es meist erforderlich, zusätzliche Informationen aus dem Umfeld des Patienten zu erfragen. Ein Gewichtsverlust von $>5 \%$ in drei Monaten beziehungsweise von $>10 \%$ in sechs Monaten ist als signifikant und prognostisch ungünstig zu werten. Existieren keine Vormessungen, können Hinweise auf $\mathrm{zu}$ weite Kleidungsstücke oder abnehmende Kleidergrößen wertvoll sein.

Ein etabliertes Maß für die Beurteilung der Ernährungssituation ist der Body Mass Index, welcher den
Quotienten aus Körpergewicht durch Körpergröße zum Quadrat darstellt. Von Untergewicht spricht man bei Erwachsenen bei einem BMI < 18,5. Dieser Wert wird nach Ansicht vieler Experten für ältere Menschen als zu niedrig angesehen. Daher ist für diese bereits ein BMI $<20$ als pathologisch zu werten.

Für Fälle von fortgeschrittener Malnutrition ist die Bestimmung des Serumalbuminspiegels ein wichtiger Prognoseindikator. In einer an 55 Patienten mit Demenz und PEG durchgeführten Studie erwies sich ein Serumalbuminspiegel unter 2,8 g/dl zum Zeitpunkt der PEG-Anlage als ein ungünstiger prognostischer Faktor bezüglich des Überlebens nach sechs Monaten (22). Nach allgemeiner Ansicht sollte bereits bei einem Albuminwert < 3,5 g/dl von einer signifikanten pathologischen Erniedrigung ausgegangen werden.

Ziel der Beurteilung des Ernährungszustandes von dementen $\mathrm{Pa}$ tienten ist es jedoch, Frühstadien der Mangelernährung beziehungsweise bereits die Gefährdung für

\section{Tab. 2 Kurzform des Mini Nutritional Assessments - MNA-sf (7)}

\section{A Hat der Patient einen verminderten Appetit?}

- Hat er während der letzten 3 Monate wegen Appetitverlust, Verdauungsproblemen, Schwierigkeiten beim Kauen oder Schlucken weniger gegessen (Anorexie)?

0 = schwere Anorexie; 1 = leichte Anorexie; 2 = keine Anorexie

B Gewichtsverlust in den letzten 3 Monaten

0 = Gewichtsverlust $>3$ kg; 1 = weiß es nicht; 2 = Gewichtsverlust zwischen 1 und 3 kg;

$3=$ kein Gewichtsverlust

C Mobilität/Beweglichkeit

0 = vom Bett zum Stuhl; 1 = in der Wohnung mobil; 2 = verlässt die Wohnung

D Akute Krankheit oder psychischer Stress während der letzten 3 Monate? $0=\mathrm{ja} ; 2$ = nein

E Psychische Situation

0 = schwere Demenz oder Depression; 1 = leichte Demenz oder Depression;

2 = keine Probleme

F Körpermassenindex (Body Mass Index, BMI)

$0=\mathrm{BMI}<19 ; 1=\mathrm{BMI} 19-21 ; 2=\mathrm{BMI} 21-23 ; 3=\mathrm{BMI}=23$

Punktwert < 11: Risiko für Mangelernährung gegeben, vollständiger MNA erforderlich

eine solche zu erkennen. Nicht zuletzt aufgrund der für die ältere Population unzureichend vorhandenen Normwerte erweist sich hierbei die Zusammenschau von klinischen, anthropometrischen, funktionellen und biochemischen Aspekten den Einzelbefunden als überlegen (30).

Hilfreich kann in diesem Sinne auch der Einsatz eines validierten Fragebogens wie des Mini Nutritional Assessment (MNA) sein. Für diesen existiert eine Kurz- und eine Langfassung. Erstere ist in wenigen Minuten durchführbar und umfasst lediglich sechs Fragen. Sie ermöglicht das Screening auf das Vorliegen einer Mangelernährung (Tab. 2). Liegt der Punktwert unter 11, gilt der Patient als gefährdet und es schließt sich die Durchführung des vollständigen MNA an, welcher die Patienten entsprechend dem nun anhand von 18 Fragen ermittelten Punktwert in drei Gruppen unterteilt:

- A: keine Gefährdung

- B: Gefährdung für Mangelernährung

- C: Mangelernährung.

Aufgrund der durch die einzelnen Fragen erhaltenen Informationen ergeben sich bereits Ansatzpunkte für eine mögliche Intervention.

Der MNA wurde in mehreren Studien erfolgreich bei Patienten mit Demenz eingesetzt $(10,16)$. Hierbei zeigte sich unter 674 älteren ambulanten psycho-geriatrischen und Alzheimer Patienten eine Häufigkeit der manifesten Mangelernährung von im Mittel $5 \%$ sowie für eine Gefährdung von $31 \%$. Für $64 \%$ ergab sich eine normale Ernährungssituation.

Die Anwendung eines solchen Fragebogens wie des MNA wird allerdings in der Regel nur in frühen bis mittleren Stadien der Demenzentwicklung möglich sein und oftmals die Hilfe von Personen aus dem Umfeld des Patienten erforderlich machen. Der Fragebogen kann kostenfrei in verschiedenen Sprachen aus dem Internet heruntergeladen werden (www.mna-elderly.com/practice/ user_guide/mna_guide.pdf).

\section{Ernährungstherapie}

Die große Umfrage der britischen Alzheimer Gesellschaft aus 
STALEVO $^{\circledR} 50 \mathrm{mg} / 12,5 \mathrm{mg} / 200 \mathrm{mg}$ Filmtabletten STALEVO ${ }^{\circledR} 100 \mathrm{mg} / 25 \mathrm{mg} / 200 \mathrm{mg}$ Filmtabletten STALEVO ${ }^{\circledR} 150 \mathrm{mg} / 37,5 \mathrm{mg} / 200 \mathrm{mg}$ Filmtabletten

Zusammensetzung: STALEVO ${ }^{\circledR} 50 \mathrm{mg} / 12,5 \mathrm{mg} / 200 \mathrm{mg}$ Arzneilich wirksame Bestandteile: $50 \mathrm{mg}$ Levodopa, $12.5 \mathrm{mg}$ Carbidopa, $200 \mathrm{mg}$ Entacapon. STALEVO 100 mg/25 mg/200 mg. Arzneilich wirksame Bestand teile: $100 \mathrm{mg}$ Levodopa, $25 \mathrm{mg}$ Carbidopa, $200 \mathrm{mg}$ Entacapon. STALEVO ${ }^{\circledR} 150$ mg/37,5 mg/200 mg. Arzneilich wirksame Bestandteile: $150 \mathrm{mg}$ Levodopa, $37,5 \mathrm{mg}$ Carbidopa, 200 mg Entacapon. Sonstige Bestandteile der 3 Dosisstärken: Croscarmellose-Natrium, Magnesiumstearat, Maisstärke, Mannitol (E421), Povidon K30 (E1201), Glycerol (E422), Hypromellose, Polysorbat 80 , Eisen(III)-oxid (E172), Sucrose, Titandioxid (E171), Eisenoxidhydrat $\mathrm{x}_{2} \mathrm{O}$ (E172). Anwendungsgebiete: STALEVO ${ }^{\circledR}$ wird bei Patienten mit Morbus Parkinson eingesetzt, bei denen „End-of-dose“-Fluktuationen im Krankheitsbild auftreten, die durch eine Behandlung mit Levodopa und einem Dopadecarboxylase-Hemmer nicht ausreichend stabilisiert sind. Gegenanzeigen: Überempfindlichkeit gegenüber Levodopa, Carbidopa Entacapon oder einem sonstigen Bestandteil von STALEVO ${ }^{\mathbb{B}}$. Engwinkelglaukom. Tumor der Nebenniere. Gleichzeitige Einnahme selektiver MAO-A- und MAO$\mathrm{B}-\mathrm{Hemmer}$ bzw. Einnahme nichtselektiver MAOHemmer. Malignes neuroleptisches Syndrom oder atraumatische Rhabdomyolyse in der Anamnese. Schwere Funktionsstörung der Leber. Patienten unter 18 Jahren. Schwangerschaft und Stillzeit. Besondere Vorsicht bei der Einnahme von STALEVO ${ }^{\circledR}$ ist erforderlich: Falls der Pat. schon einmal eine Herzattacke, Asthma oder andere Erkrankungen des Herzens, der Blutgefäße oder der Lunge gehabt hat. Falls Probleme mit der Leber vorliegen; die Dosis muss möglicherweise angepasst werden. Falls der Pat. schon einmal eine Erkrankung der Nieren, der Leber, eine Hormonstörung, ein Magengeschwür, Krampfanfälle oder eine schwere psychische Erkrankung gehabt hat. Falls der Pat sich depressiv verstimmt fühlt, Selbstmordgedanken hegt oder untypische Verhaltensänderungen an sich bemerkt Falls ein chronisches Weitwinkelglaukom vorliegt; die Dosis muss möglicherweise angepasst und der Augeninnendruck überwacht werden. Falls andere Arzneimitte eingenommen werden, die den Blutdruck senken können; STALEVO ${ }^{\circledR}$ kann diese Reaktion verstärken. Falls es bei dem Patienten zu plötzlich einsetzenden Schlafepisoden kommt oder er starke Schläfrigkeit verspürt Falls es nach Einleitung der Behandlung mit STALEVO zu unwillkürlichen Bewegungen kommt oder sich diese verschlimmern. Falls sich schwere Muskelsteifigkeit, starke Muskelzuckungen, Zittern, Erregung, Verwirrtheit, Fieber, beschleunigter Herzschlag oder erhebliche Schwankungen des Blutdrucks einstellen. Falls die Behandlung mit STALEVO ${ }^{\circledR}$ abgebrochen werden muss, sollte STALEVO ${ }^{\circledR}$ möglichst stufenweise abgesetzt und die weitere Antiparkinson-Medikation angepasst werden. Nebenwirkungen: Die häufigsten Nebenwirkungen sind: unwillkürliche Bewegungen, Übelkeit, psychische Veränderungen einschließlich paranoider und psychotischer Symptome, Depression (möglicherweise mit Selbstmordgedanken) sowie Probleme mit dem Erinnerungsvermögen oder gezieltem Nachdenken, harmlose rötlich-braune Verfärbung des Urins. Wenige häufige Nebenwirkungen sind: unregelmäßiger Herzschlag und Herzrhythmus; Schwindel oder Ohnmacht aufgrund niedrigen Blutdrucks; Benommenheit; ungewöhnliche Schläfrigkeit; plötzliche Verschlimmerung von Parkinson-Symptomen; Appetitverlust; Erbrechen Blutungen im Magen-Darm-Trakt; Entwicklung eines Zwölffingerdarmgeschwürs; Bauchschmerzen; Mundtrockenheit; Verstopfung; Durchfall; Bluthochdruck Entzündung der Beinvenen; Schlaflosigkeit; Halluzinationen; Verwirrtheit, unangenehme Träume; Müdigkeit; Muskelkrämpfe; vermehrtes Schwitzen; häufigere Stürze; Veränderungen der Blutzellen und ihrer Bestandteile, was zu Müdigkeit, Ohnmacht, Infektionen führen kann. Blutungen; Brustschmerzen; Kurzatmigkeit; Kribbeln oder Taubheitsgefühl; Krampfanfälle. Seltene oder sehr seltene Nebenwirkungen sind: Erregung, Juckreiz und Hautausschläge Abnahme oder Zunahme des Körpergewichts, Sehstörungen. Verschreibungspflichtig Zulassungsinhaber: Orion Corporation, Orionintie 1 FIN-02200 Espoo, Finnland. Für Informationen zu diesem Arzneimittel wenden Sie sich bitte an den lokalen Vertreter des Zulassungsinhabers: Orion Pharma GmbH Notkestraße 9, D-22607 Hamburg, Tel: 040/89 96 89-0. Handelsformen: 30 Filmtabletten (N1), 100 Filmtabletten (N3), Stand der Information: Oktober 2003 dem Jahr 2000 zeigte, dass viele Betroffene in Heimen und Krankenhäusern nicht genug zu trinken hatten, dass für sie keine ausreichende Auswahl an Speisen vorhanden war, und dass man ihnen nicht genug Zeit zum Essen gab oder ihnen keine Hilfe bei den Mahlzeiten angeboten wurde. 33\% hatten Probleme mit dem Schlucken und 46\% machten sich Sorgen über geringen Appetit und Gewichtsverlust. Die meisten der Patienten wussten jedoch nicht, an wen sie sich mit ihren Problemen und Sorgen wenden sollten (2).

Diese Erhebung verdeutlicht, dass sich die Diskussion von Ernährungsschwierigkeiten bei Dementen nicht auf den Einsatz von Supplementen (Nahrungsergänzungsmittel) oder enteraler Ernährung konzentrieren darf, sondern dass auch der natürlichen Nahrungsaufnahme von Dementen in frühen und mittleren Krankheitsstadien genügend Aufmerksamkeit im Alltag von Institutionen geschenkt werden muss.

Mehrere Arbeiten der letzten Jahre zeigten dabei, dass ein rasch progredienter Gewichtsverlust bei Alzheimerpatienten nicht zwingend und eine Umkehr der Gewichtsentwicklung möglich ist.

\section{Allgemeine Ansatzpunkte}

Bei Patienten in frühen Krankheitsstadien ist Hilfe beim Einkaufen sowie bei der Vorbereitung und dem Kochen von Speisen ein wertvoller Ansatz. Auch in fortgeschrittenen Stadien kann die Einbeziehung der dementen Patienten in die Speisezubereitung und das Kochen das Verhältnis zur Nahrungsaufnahme verbessern und ihnen auch unabhängig hiervon Freude bereiten. Entsprechende Angebote sind insbesondere in Rehabilitationseinrichtungen und Tageskliniken wünschenswert.

In unserer heutigen multikulturellen Gesellschaft bedürfen ferner tradierte kulturelle Aspekte der Ernährung innerhalb verschiedener ethnischer Gruppen der besonderen Beachtung.

Ein angenehmes und vertrautes Ambiente während der Mahlzeiten scheint sich günstig auf den Umfang der Nahrungsaufnahme auszuwirken. So zeigen die Ergebnisse ver- schiedener Studien, dass auch ungewöhnliche Ansätze erfolgreich sein können. Edwards und Beck untersuchten die Wirkung, die das Aufstellen von Aquarien in den Speisesälen auf die Nahrungsaufnahme bei 62 dementen Heimbewohnern entfaltete. Diese Arbeit erstreckte sich über 16 Wochen. In der Kontrollgruppe wurde im Speisesaal lediglich eine Fototapete mit Meeresblick angebracht. In der Therapiegruppe, in deren Speiseraum die Aquarien aufgestellt waren, ließ sich am Ende der Beobachtungszeit ein signifikanter Gewichtszuwachs beobachten. Die Autoren vermuten, dass die Heimpatienten mehr aßen, da sie für längere Zeiten bei Tisch saßen, um den Fischen zuzuschauen, statt umherzuwandern. Lethargische Patienten wurden möglicherweise durch die Fische angeregt und waren dem Essen gegenüber aufmerksamer. Die Einnahme von Supplementen sank in der Therapiegruppe um 25\% (12).

Das Essen soll frisch und appetitlich präsentiert werden. Auch kann stärkeres Würzen der Speisen und der Einsatz von Geschmacksverstärkern zu einer Steigerung der Nahrungsaufnahme beitragen.

In fortgeschrittenen Stadien der Demenzentwicklung lassen sich durch eine angepasste Verarbeitung der Nahrungskomponenten günstige Ergebnisse erzielen. Für Patienten mit Schluckstörungen empfiehlt sich eine Auswahl an Speisen mit veränderter Konsistenz. Generell sollte das Nahrungsangebot für demente Patienten auch sogenanntes Fingerfood einbeziehen, welches den Einsatz von Besteck überflüssig macht. Zwischen den Hauptmahlzeiten sollten zusätzlich Snacks angeboten werden. Auch ist oftmals der Verzehr von „gesundem Essen“ im strengen Sinne bei dieser Patientengruppe zweitrangig, wenn überhaupt ausreichend gegessen wird. Biernacki und Mitarbeiter erzielten mit einem Konzept, das diese Grundsätze beachtete, bei einer Patientengruppe mit einem Mini Mental Score < 3 über einen Zeitraum von sechs Jahren einen Anstieg des BMI von 19 auf 25 (5).

Es liegen nur wenige wissenschaftliche Untersuchungen zur 
Hilfe von Pflegepersonal bei der Nahrungsaufnahme (Verabreichung pürierter Kost mit Hilfe von Spritzen, Löffeln, Hilfe beim Zerkleinern oder Bewegen von Speiseteilen) vor. Es gilt jedoch zu bedenken, dass diese Form der Ernährung als wesentliche Komponente persönliche Zuwendung beinhaltet. Essen bzw. Mahlzeiten sind kleine soziale und oft auch kommunikative Ereignisse, die Freude am Leben vermitteln können und bezüglich ihrer sozialen Wertigkeit nicht zu ersetzen sind. Die Einbeziehung von Angehörigen in diese Funktionen kann helfen, personelle Engpässe im Bereich der professionellen Pflege zu lindern und den sozialen Aspekt gleichzeitig stärken.

Auch sind Schwestern und Fürsorgende im Umfeld des Patienten gezwungen, die emotionellen Aspekte zu reflektieren, denen sie bei ihrer Hilfe während der Nahrungsaufnahme bei Dementen begegnen. Es bedarf hierbei oftmals einer bewussten Anstrengung, bestimmte Verhaltensweisen des Dementen wie das Offenhalten des Mundes, Ausspucken oder das Wegdrehen des Kopfes während des Essens nicht als grundsätzliche Ablehnung zu begreifen.

Bei den Patienten, für die mit frisch zubereiteten Speisen keine ausreichende Nahrungszufuhr mehr möglich ist, sollte der Einsatz von Trinksupplementen zur Stabilisierung der Gewichtsentwicklung erwogen und gegebenenfalls nicht $\mathrm{zu}$ lange hinausgezögert werden. In einer schwedischen Studie, welche den Einsatz von Supplementen in Kombination mit einer Ernährungsschulung der Betreuer testete, erwiesen sich lediglich erstere als dauerhaft effektiver Therapieansatz bezüglich einer Gewichtszunahme. Für die Schulung der Betreuer allein ließ sich keine dauerhafte Wirksamkeit nachweisen (13).

Die Gabe von oralen Supplementen in einer Tagesmenge zwischen 400 und 600 kcal erbrachte in zwei kontrollierten und einer weiteren offenen Studie jeweils eine signifikante Gewichtszunahme $(8,13,28)$.

Entscheidet man sich zur Therapie mit Supplementen, sollte ein
Ernährungsplan erstellt und diese zwischen den Mahlzeiten verabreicht werden. Andernfalls könnte es bei zu großer Nähe zu den Hauptmahlzeiten zu einem Appetitverlust bzw. zu einer vorzeitigen Sättigung kommen.

\section{- Enterale Ernährung}

Vor der Einleitung einer enteralen Ernährung müssen intensive Anstrengungen unternommen werden, eine ausreichende Nahrungs- und Flüssigkeitszufuhr auf oralem Wege zu erreichen. Dies schließt auch eine Beurteilung der Sprech- und Schluckfähigkeit mit Hilfe logopädischer und radiologischer Verfahren ein.

Zusätzlich sind verschiedene Aspekte für eine richtige Indikationsstellung zur enteralen Ernährung zu berücksichtigen:

- der Patientenwille bezüglich einer enteralen Ernährung bzw. der mutmaßliche Wille, falls eine angemessene persönliche Willensbekundung zum betreffenden Zeitpunkt nicht mehr möglich ist

- die Schwere der Grunderkrankung

- die individuelle Lebenserwartung des Betroffenen

- seine zu erwartende Lebensqualität mit und ohne enterale Ernährung

- eventuell zu erwartende Komplikationen und Beeinträchtigungen im Rahmen der enteralen Ernährung.

Die Entscheidung bezüglich der Durchführung einer enteralen Ernährung bedarf immer einer differenzierten Beurteilung des Einzelfalles. In diese Entscheidungsfindung müssen die Angehörigen, falls vorhanden, der gesetzliche Betreuer, die Pflegekräfte, die behandelnden Therapeuten und Ärzte einbezogen werden. In Zweifelsfällen ist das Vormundschaftsgericht einzuschalten.

Eine enterale Ernährung kann mittels dreier unterschiedlicher Verfahren verabreicht werden: die Anlage einer nasogastrischen Sonde, die perkutane Gastrostomie (PEG) sowie die chirurgische Jejunostomie. Erstere ist nur für eine temporäre Nahrungszufuhr im Bereich von Ta- gen und wenigen Wochen geeignet. PEG und chirurgische Jejunostomie hingegen eignen sich für eine unbefristete enterale Nahrungszufuhr, wobei die Indikation zu letzterer bei fehlendem endoskopischen Zugang zum Magen/Jejunum oder bei Unmöglichkeit der perkutanen Punktion gestellt wird.

Kommt es im Rahmen einer akuten Erkrankung eines Demenzpatienten $\mathrm{zu}$ einer deutlichen Verschlechterung der oralen Nahrungsaufnahme, sollte vor Durchführung einer PEG die Anlage einer nasogastrischen Sonde erwogen werden. Diese ist für eine kurzfristige enterale Ernährung ein geeignetes Mittel und kann bei Besserung der Nahrungsaufnahme ohne Aufwand wieder gezogen werden. Zusätzlich gilt es zu berücksichtigen, dass eine beträchtliche Zahl der betroffenen Patienten eine akute Verschlechterung ihres Gesundheitszustandes nicht überlebt.

Zwar findet sich für die PEG eine niedrige Komplikationsrate zwischen $0-2 \%$. Dennoch liegt in mehreren Arbeiten die perinterventionelle Mortalität zwischen 6-28\%. Dies ist in erster Linie durch die Grunderkrankung und die Komorbidität der Patienten bedingt.

Eine Auswertung von 361 konsekutiven Patienten aus Großbritannien, bei denen PEGs gelegt wurden, erbrachte für die Untergruppe der Demenzkranken eine höhere Mortalitätsrate als sie in der Gruppe der Nicht-Demenzkranken zu beobachten war. Die 30-Tage Mortalität lag für die Demenzkranken bei $54 \%$, die 1-Jahresmortalität bei $90 \%$ gegenüber $28 \%$ und $63 \%$ in der Gesamtgruppe (27). Sollte sich der Zustand des Patienten im Verlauf stabilisieren und die orale Nahrungszufuhr weiterhin unzureichend sein, ist die Indikation zur PEG erneut zu diskutieren.

Weder für die PEG noch für die Jejunostomie konnten bislang eine Verminderung des Auftretens von Aspirationspneumonien gegenüber oraler Ernährung nachgewiesen werden. Pick und Mitarbeiter zeigten für Heiminsassen hingegen, dass sowohl das Vorliegen einer nasogastralen Sonde als auch eine PEG prädisponierende Faktoren für das Auftreten von 
Aspirationen darstellen können (23). In Kenntnis dieser Tatsache ist besonderer Wert auf die $30^{\circ}$-Oberkörperhochlagerung des Patienten zu legen. Auch sollte bei persistierenden Problemen an den Einsatz einer Ernährungspumpe gedacht werden.

Die bislang vorliegenden Arbeiten zur enteralen Ernährung mittels PEG bei Demenz sind in ihren Ergebnissen oftmals widersprüchlich. So gelang bei Durchführung einer Sondenernährung in zwei Studien bei hochbetagten dementen Bewohnern von Pflegeheimen eine signifikante Gewichtszunahme $(15,24)$. Diese war in der zweiten Arbeit nach sechs Monaten bei $91 \%$ der enteral Ernährten zu beobachten und lag im Mittel bei 5,4 $\mathrm{kg} \pm 4,4 \mathrm{~kg}$. In zwei weiteren Studien konnte jedoch keine Zunahme des Körpergewichts, aber ein Anstieg des Albuminspiegels erzielt werden ( 7 , 11). In mehreren der erwähnten Studien wurde der funktionelle Status der Patienten vor und während der enteralen Ernährung erfasst. Eine diesbezügliche Verbesserung konnte nicht nachgewiesen werden.

Es liegen gegenwärtig vier Studien vor, die zur Beantwortung der Frage, ob eine enterale Ernährung bei Patienten mit fortgeschrittener Demenz eine Verlängerung des Überlebens ermöglicht, herangezogen werden können $(18,20,21,26)$. Die Zahl der eingeschlossenen Patienten pro Studie betrug zwischen 41 und 1386. Diese Arbeiten konnten nach Beobachtungszeiträumen zwischen zwei und zwölf Monaten keinen signifikanten Unterschied in der Überlebensrate zwischen der enteral gegenüber der oral ernährten Gruppe nachweisen. Lediglich die Datenbankanalyse von Rudberg (26) konnte nach 30 Tagen eine auf die Hälfte reduzierte Mortalität in der enteral ernährten Gruppe dokumentieren. Diese lag bei $15 \%$. Auch nach einem Jahr war ein signifikanter, wenngleich geringer Unterschied zwischen den beiden Therapiegruppen erhalten. Nach Angaben der Autoren waren in dieser Studie die beiden Gruppen bezüglich Demenzgrad, Komorbiditäten, funktionellem Status und BMI vergleichbar.

Generell leidet die Wertigkeit der Studiendaten zum Thema enterale
Ernährung und Demenz an der Heterogenität der untersuchten Patientenpopulation sowie bei Vergleich einer enteral via Sonde oder PEG ernährten Gruppe mit einer oral ernährten Gruppe an der meist fehlenden Randomisation. Neben hohen Mortalitätszahlen wurde in anderen Arbeiten ein langes Überleben beobachtet. Diese Unterschiede dürften wesentlich in den unterschiedlichen Komorbiditäten sowie in den verschiedenen Stadien der Demenzerkrankung begründet sein. So zeigten mehrere Analysen, dass Schluckschwierigkeiten und Schluckunfähigkeit einen unabhängigen Risikofaktor für die Mortalität von Demenzkranken darstellen und dies unabhängig vom Vorliegen einer PEG ist $(9,14)$.

Aufgrund der vorliegenden Studienergebnisse kommt die Expertenkommission, welche unter der Schirmherrschaft der Deutschen Gesellschaft für Ernährungsmedizin (DGEM) und der Deutschen Gesellschaft für Geriatrie (DGG) die Leitlinie Enterale Ernährung in der Geriatrie verfasste, $z u$ dem Resumee, dass sich die Frage nach einer Lebensverlängerung durch eine enterale Sondenkost gegenwärtig nicht zufriedenstellend beantworten lässt (29).

Da die Prognose bei über Sonde ernährten Demenzpatienten sehr unterschiedlich sein kann, wird jedoch in frühen und mittleren Krankheitsstadien eine ausreichende und hochwertige Ernährung empfohlen. Für fortgeschrittene Demenzstadien wird mit Verweis auf die vorliegende Datenlage keine Empfehlung für eine Sondenernährung ausgesprochen. Die Anlage einer PEG soll in diesen Fällen immer eine Einzelentscheidung sein. Für final Demenzkranke wird eine Sondenernährung nicht empfohlen.

\section{Ethische Aspekte}

Ein klassisches pflegerisches und ethisches Dilemma in Bezug auf die Nahrungszufuhr bei Dementen ist die Frage, ob durch die Nahrungszufuhr für den Betroffenen eine Stresssituation entsteht, oder ob durch das Sistieren einer adäquaten Nahrungszufuhr eine Malnutrition mit all ihren Konsequenzen erst induziert wird. Man nennt dies den „double-blind conflict“"(1). Dieser sollte immer wieder in Teambesprechungen oder mit betreuenden Angehörigen thematisiert werden. Es ist dies auch einer der Momente, in denen divergente Meinungen bezüglich der Anlage einer PEG offenkundig werden können.

Die PEG ist somit - 20 Jahre nach ihrer Einführung - zu einer Art Metapher in Bezug auf die Sinnhaftigkeit von Pflegemaßnahmen bei Demenzkranken geworden. Die Datenlage (siehe oben) ist nach wie vor ungenügend. Dies nicht nur im Hinblick auf klassische „outcome data“, sondern auch bezüglich eines interdisziplinären Diskurses. Dabei sollte die Symbolik der PEG nicht außer Acht gelassen werden; rein optisch imponiert sie ja als eine wieder implementierte Nabelschnur.

Fragen zu ethischen Überlegungen zu einer an Demenzkranke adaptierten Therapie müssen deshalb künftig auch im Bereich der Nahrungszufuhr interdisziplinär thematisiert werden. Gerade auch ein mehr „palliativ“ orientierter Betreuungszugang bei Betagten - und demente Menschen sind in der großen Mehrheit betagt - mag in dreierlei Hinsicht die momentan vorherrschenden bioethischen Sichtweisen bereichern:

- Die mehr palliativ orientierte Pflege gibt ein Gegengewicht zur vorherrschenden rein bioethischen Sichtweise. Eine interpretative Ethik orientiert sich hier mehr an den bestehenden Versorgungsstrukturen und den Bedürfnissen Betroffener und ihrer Umgebung

- $\quad$ Eine mehr begleitende Medizin zeigt die Einseitigkeit heutiger moralischer Prinzipien auf, nämlich die der Autonomie. Der Betroffene muss nicht nur im Hinblick auf sein ihm eigenes Leiden gesehen werden, sondern auch als Mitglied einer näheren persönlichen Umgebung, die ihn betreuen kann

- Eine geriatrisch orientierte Palliativmedizin erinnert auch an gewisse menschliche Spezifika, die in einer postmodernen Gesellschaft gerne vergessen werden: Den (dementen) Menschen mehr als Bürger denn als Konsument zu sehen. 
In diesem Kontext spielt die Ernährung eine weit größere Rolle als das alleinige Zuführen von Nährstoffen. Hilfsbedürftige - auch aus einer persönlichen Hilflosigkeit heraus - zu „laben“, ist tief in allen Gesellschaften verankert. „Man ist, solange man isst“ ist demzufolge keine leere Worthülse, sondern tangiert Grundfragen des Lebens, gerade auch in der Interaktion mit Dementen, wo andere Sinnesreize krankheitsbedingt ins Leere zielen.

Hier die richtigen Entscheidungen zu treffen, kommt einer Gratwanderung gleich, in der die Routen immer wieder neu adjustiert werden müssen und sollen. Fachpersonen im Gesundheitswesen mögen hier als Tourenführer ihre Funktion haben, dennoch ist es die gesamte Seilschaft (sinnbildhaft für die betroffene Gesellschaft), die die Marschroute bestimmen soll und hoffentlich in Zeiten einer oft zu stark rein bioethisch bestimmten Gesundheitspolitik auch weiter bestimmen darf.

\section{Nutrition and Dementia}

As a consequence of the actual trends in demography the number of patients with dementia will steadily increase during the next decades. One of the most important problems in caring for these patients is to provide adequate nutrition during the different phases of this disease. On the one hand it is essential to avoid early malnutrition which may cause further functional impairment. On the other hand one is to withhold nutritional overtherapy in the final stages of dementia. Patients with dementia should be screened for malnutrition in the early and middle stages of this disease. For patients with risk of or with overt malnutrition individual nutritional plans should be provided. Enteral nutrition in advanced stages of dementia should only be untertaken after a careful and individualized analysis of the situation.

\section{Keywords:}

dementia - nutritional screening enteral nutrition - percutaneous endoscopic gastrostomy - PEG ethics

\section{Literatur}

1. Akerlund BM, Norberg A. An ethical analysis of double blind conflicts as experienced by careworkers feeding severely demented patients. Int J Nursing Studies 1985; 22: 207-216

2. Alzheimer's Society. Food for thought. London: Alzheimer's Society, 2000

3. Andrieu $S$ et al. Nutritional risk factors for institutional placement in Alzheimer's disease after one year follow-up. J Nutr Health Aging 2001; 5: 113-117

4. Balestreri L, Grossberg A, Brossberg GT. Behavioral and psychological symptoms of dementia as a risk factor for nursing home placement. Int Psychogeriatr 2001; 12 (Suppl 1): 59-62

5. Biernacki C, Barratt J. Improving the nutritional status of people with dementia. Br J Nursing 2001; 10: 1104-1114

6. Burns BJ et al. Mental health service use by the elderly in nursing homes. Am J Public Health 1993; 83: 331

7. Callahan $\mathrm{CM}$ et al. Outcomes of percutaneous endoscopic gastrostomy among older adults in a community setting. J Am Geriatr Soc 2000; 48: 1048-1054

8. Carver AD, Dobson AM. Effects of dietary supplementation of elderly hospital residents. J Hum Nutr Diet 1995; 8: 389-394 9. Cefalu CA. Appropriate dysphagia evaluation and managment of the nursing

home patient with dementia. Ann Long Term Care 1999; 7: 447-451

10. De Mendonca Lima CA et al. Nutritional assessment in patients attending a psychogeriatric hospital. Age and Nutrition 1999; 10: 9-13

11. Dwolatzky T et al. A prospective comparison of the use of nasogastric and percutaneous endoscopic gastrostomy tubes for long-term enteral feeding in older people. Clin Nutr 2001; 20: 535-540

12. Edwards NE, Beck AM. Animal assisted therapy and nutrition in Alzheimer's disease. West J Nursing Res 2002; 24: 697-712

13. Faxen-Irving $G$ et al. The effect of nutritional intervention in elderly subjects residing in group-living for the demented. Eur J Clin Nutr 2002; 56: 221-227

14. Gillick MR. Rethinking the role of tube feeding in patients with advanced dementia. N Engl J Med 2000; 20: 206-210

15. Golden $A$ et al. Long-term survival of elderly nursing home residents after percutaneous endoscopic gastrostomy for nutritional support. Nurs Home Med 1997; 5: 382-389

16. Guigoz Y, Lauque S, Vellas BJ. Identifying the elderly at risk for malnutrition - The Mini Nutritional Assessment. Clin Geriatr Med 2002; 18: 737-757

17. Heston LL, Gerrard J, Makris L. Inadequate treatment of depressed nursing home elderly. J Am Geriatr Soc 1992; 40: 117-122

18. Meier DE et al. High short-term mortality in hospitalized patients with advanced dementia: lack of benefit of tube feeding. Arch Intern Med 2001; 161: 594-599

19. Miller DK et al. Abnormal eating attitudes and body image in older undernouris- hed individuals. J Am Geriatr Soc 1991; 39: 462

20. Mitchell SL, Kiely DK, Lipsitz LA. The risk factors and impact on survival of feeding tube placement in nursing home residents with severe cognitive impairment. Arch Intern Med 1997; 157: 327-332

21. Murphy LM, Lipman TO. Percutaneous endoscopic gastrostomy does not prolong survival in patients with dementia. Arch Intern Med 2003; 163: 1351-1353

22. Nair S, Hretan H, Pitchumoni CS. Hypoalbuminemia is a poor predictor of survival after percutaneous endoscopic gastrostomy in elderly patients with dementia. Am J Gastroenterol 2000; 95: 133-136

23. Pick $\mathrm{N}$ et al. Pulmonary aspiration in a long-term care setting: Clinical and laboratory observations and an analysis of risk factors. J Am Geriatr Soc 1996; 44: 763-768

24. Peck A, Cohen Ce, Mulvihill MN. Longterm enteral feeding of aged demented nursing home patients. J Am Geriatr Soc 1990; 38: 1195-1198

25. Rovner BW, German PS, Broadhead J. The prevalence and management of dementia and other psychiatric disorders in nursing homes. Int Psychogeriatr 1990; 2: 13-24

26. Rudberg MA et al. Effectiveness of feeding tubes in nursing home residents with swallowing disorders. J Parent Ent Nutr 2000; 24: 97-102

27. Sanders DS et al. Survival analysis in percutaneous endoscopic gastrostomy feeding: A worse outcom in patients with dementia. Am J Gastroenterol 2000; 95: 1472-1475

28. Wouters-Wesseling $W$ et al. Study of the effect of a liquid nutrition supplement on the nutritional status of psycho-geriatric nursing home patients. Eur J Clin Nutr 2002; 56: 245-251

29. Volkert D et al. Leitlinie Enterale Ernährung in der Geriatrie und geriatrisch-neurologischen Rehabilitation der Deutschen Gesellschaft für Ernährungsmedizin und der Deutschen Gesellschaft für Geriatrie e.V. Aktuel Ernaehr Med 2004; 29: 1-28

30. Volkert D. Ernährungszustand, Energieund Substratstoffwechsel im Alter. Aktuel Ernaehr Med 2004; 29: 19-197

\section{Korrespondenzadresse:}

Dr. Jürgen Bauer

Institut für Biomedizin des Alterns der FAU Erlangen-Nürnberg

Med. Klinik II - Klinikum Nürnberg

Heimerichstr. 58

90419 Nürnberg

E-Mail: bauer_j@klinikum-nuernberg.de 